\title{
Modal Density of Honeycomb Sandwich Composite Cylindrical Shells Considering Transverse Shear Deformation
}

\author{
S. Josephine Kelvina Florence and K. Renji \\ ISRO Satellite Center, ISRO Vimanapura Post, Bangalore, India 560017.
}

\author{
K. Subramanian \\ P.S.R. Engineering College, Sivakasi, India 626104.
}

(Received 23 May 2016; accepted 17 November 2016)

\begin{abstract}
Honeycomb sandwich shells with composite face sheets are of extensive use in the spacecraft industry. Information on the number of resonant modes present in a frequency band is required to study their response behaviour under acoustic excitation. Modal densities of thin composite cylindrical shells have been reported while transverse shear deformations have not been considered. But in honeycomb sandwich panels, transverse shear deformations are significant, especially at higher order modes of vibration. In this work expressions for natural frequency and modal density of composite cylinders incorporating transverse shear deformation are derived. The modal densities of a typical cylinder that are obtained using the derived expression are compared with the results obtained using the finite element method and they are similar. Effects of transverse shear and orthotropic nature of the face sheets on the modal densities are investigated. It is shown that computing the modal density of honeycomb sandwich cylinders without considering the transverse shear deformation can lead to significant errors at high frequencies. Expressions of modal densities for special cases are also derived from the general expression.
\end{abstract}

\section{NOMENCLATURE}

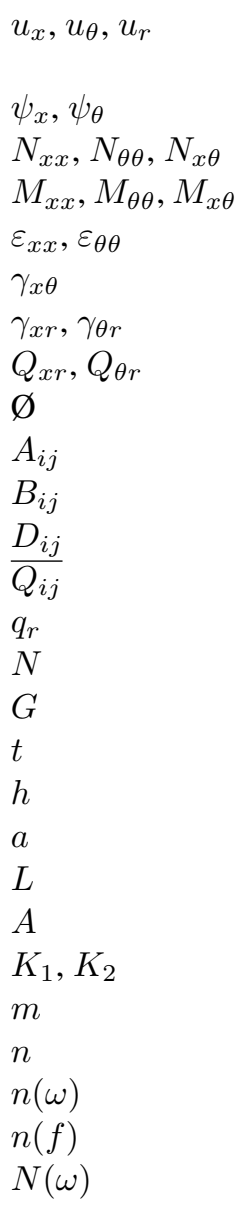

$N(f)$ displacement along the longitudinal, tangential and radial directions

rotations of the transverse plane

force resultants per unit length

moment resultants per unit length

in-plane direct strains

in-plane shear strain

transverse shear strains

shear forces per unit length

Airy's stress function

extensional stiffness terms

coupling stiffness terms

bending stiffness terms

coefficients of elastic stiffness

external transverse force per unit area

shear rigidity of the shell

shear modulus of the core

thickness of face sheet

thickness of the core

radius of the cylinder

length of the cylinder

Area of the cylinder

function of wave numbers

axial half wave number

circumferential full wave number

number of modes per rad/s

number of modes per $\mathrm{Hz}$

number of modes below the radian frequency ' $\omega$ '

number of modes below the cyclic frequency ' $f$ '

\section{INTRODUCTION}

Honeycomb sandwich plates/shells are widely used in satellite structures due to their high stiffness to weight ratio. Broad band acoustic excitation is one of the critical loading conditions for satellite structures. Statistical Energy Analysis (SEA) is a promising tool to study the response behaviour of structures subjected to such high frequency dynamic loads. Modal density is an important parameter encountered in the calculation of response using SEA. ${ }^{1,2}$ Expressions for modal densities of several structural forms are derived and are in use. Xie et al. ${ }^{3}$ presented mode counts of several simple structural systems with various basic boundary conditions. They confirmed that at high frequencies the modal density is independent of boundary conditions.

In several larger spacecrafts, the primary structure consists of a central cylinder which is a honeycomb sandwich construction with composite face sheets. Several studies have been reported on modal densities of cylindrical shells. Modal densities of honeycomb sandwich cylinders have also been reported. Wilkinson ${ }^{4}$ derived an expression for modal densities of sandwich cylinders incorporating shear deformation of the core and Erickson ${ }^{5}$ modified the expression considering rotary inertia. Ferguson and Clarkson ${ }^{6}$ obtained an expression for estimating modal density of paraboloidal structural element. Elliot ${ }^{7}$ presented expressions for the modal densities of thin as well as honeycomb sandwich cylindrical shells in the form of integrals which were evaluated numerically. Finnvedan ${ }^{8}$ presented a finite element based approach to estimate the modal density of shells and hence no expressions are derived and it was applied to isotropic shells. An alternate methodology to evaluate the modal density of circular cylindrical shells is presented by Farshidianfar et al. ${ }^{9}$ but it is applicable to only isotropic shells. Ramachandran and Narayanan ${ }^{10}$ studied the effect of stiffeners on the modal density of isotropic cylindrical shells. All the 
above works are on the isotropic shells or honeycomb sandwich shells with isotropic face sheets.

An expression for the modal densities composite cylindrical shells was derived earlier ${ }^{11}$ which can be used for estimating the modal densities of thin composite cylindrical shells. But the modal density determined using this expression does not consider the transverse shear flexibility. In honeycomb sandwich structures the shear modulus of the core is considerably low and can have a significant effect on the modal density. Therefore, an expression for modal density of cylindrical shells considering the shear flexibility is essential, especially for determining the modal densities of honeycomb sandwich composite cylinders.

In this work, modal densities of honeycomb sandwich cylindrical shells with composite face sheets are obtained. An expression for modal density of composite cylindrical shells considering transverse shear deformation is derived. An expression for natural frequency is required to derive the expression for modal density. No closed form expressions are presented in literature for estimation of natural frequencies of such shells incorporating transverse shear. Therefore, an expression for natural frequency is first derived. Expression for modal density is then derived by adopting wave space integration technique. Modal densities of a typical composite cylinder computed using the expression derived here are compared with the results obtained using the finite element method. Influence of transverse shear deformation on the modal densities is investigated. The expressions for some special cases are also presented.

\section{DIFFERENTIAL EQUATIONS OF MOTION}

Consider a cylinder having a radius $a$, length $L$ and mass per unit area of $\rho_{m}$. The co-ordinate axes are denoted by $x$ for longitudinal, $\theta$ for tangential and $\mathrm{r}$ for radial as shown in Fig. 1. The displacement along the longitudinal direction is $u_{x}$, along the tangential direction (linear displacement) is $u_{\theta}$ and along the radial direction is $u_{r}$.

In this work, Donnell's shell theory incorporating first order shear deformation along with Airy's stress function is used.

\subsection{Force and Moment Equilibrium Equations}

For laminated cylindrical shells under free vibration, neglecting rotary inertia,

$$
\begin{aligned}
\frac{\partial N_{x x}}{\partial x}+\frac{1}{a} \frac{\partial N_{\theta x}}{\partial \theta} & =\rho_{m} \frac{\partial^{2} u_{x}^{0}}{\partial t^{2}} \\
\frac{\partial N_{x \theta}}{\partial x}+\frac{1}{a} \frac{\partial N_{\theta \theta}}{\partial \theta}+\frac{Q_{\theta r}}{a} & =\rho_{m} \frac{\partial^{2} u_{\theta}^{0}}{\partial t^{2}} \\
\frac{\partial Q_{x r}}{\partial x}+\frac{1}{a} \frac{\partial Q_{\theta r}}{\partial \theta}-\frac{N_{\theta \theta}}{a} & =\rho_{m} \frac{\partial^{2} u_{r}}{\partial t^{2}} \\
\frac{\partial M_{x x}}{\partial x}+\frac{1}{a} \frac{\partial M_{\theta x}}{\partial \theta}-Q_{x r} & =0 ; \\
\frac{\partial M_{x \theta}}{\partial x}+\frac{1}{a} \frac{\partial M_{\theta \theta}}{\partial \theta}-Q_{\theta r} & =0 ;
\end{aligned}
$$

which is a set of coupled equations with 5 displacement components $\left(u_{x}^{0}, u_{\theta}^{0}, u_{r}, \psi_{x}, \psi_{\theta}\right) .{ }^{12-14}$ In the above equations $N_{x x}$, $N_{\theta \theta}, N_{x \theta}, M_{x x}, M_{\theta \theta}$, and $M_{x \theta}$ are the force and moment resultants (per unit length) and $Q_{x r}$ and $Q_{\theta r}$ are the shear forces per unit length. In the above equations $u_{x}^{0}$ and $u_{\theta}^{0}$ are the midsurface displacements and $u_{r}$ is the radial displacement. Com-

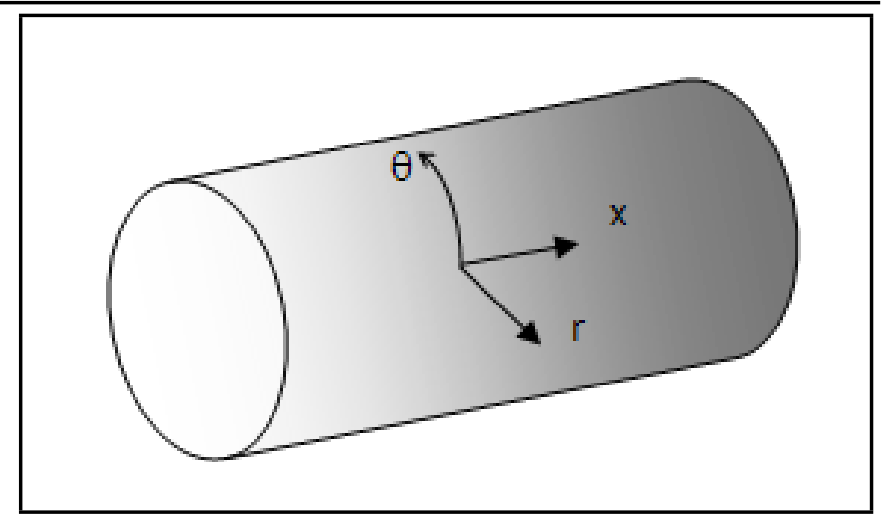

Figure 1. Coordinate system.

bining Eqs. (3), (4), and (5) we get

$$
\frac{\partial^{2} M_{x x}}{\partial x^{2}}+\frac{2}{a} \frac{\partial^{2} M_{\theta x}}{\partial x \partial \theta}+\frac{1}{a^{2}} \frac{\partial^{2} M_{\theta \theta}}{\partial \theta^{2}}-\frac{N_{\theta \theta}}{a}=\rho_{m} \frac{\partial^{2} u_{r}}{\partial t^{2}} .
$$

Considering only the normal loading and neglecting the inertia in the in-plane directions, Eq. (1) becomes

$$
\frac{\partial N_{x x}}{\partial x}+\frac{1}{a} \frac{\partial N_{\theta x}}{\partial \theta}=0
$$

Further, since the shear term $\frac{Q_{\theta r}}{a}$ is small relative to the other terms of Eq. (2), Eq. (2) reduces to

$$
\frac{\partial N_{x \theta}}{\partial x}+\frac{1}{a} \frac{\partial N_{\theta \theta}}{\partial \theta}=0
$$

Equations (6), (7), and (8) form the force and moment equilibrium equations. It is to be noted that these differential equations do not change whether the transverse shear deformation is considered or not.

\subsection{Strain Displacement Relations}

The strains are related to displacements as

$$
\begin{aligned}
\varepsilon_{x x} & =\frac{\partial u_{x}}{\partial x} \\
\varepsilon_{\theta \theta} & =\frac{1}{a} \frac{\partial u_{\theta}}{\partial \theta}+\frac{u_{r}}{a} \\
\gamma_{x \theta} & =\frac{\partial u_{\theta}}{\partial x}+\frac{1}{a} \frac{\partial u_{x}}{\partial \theta} .
\end{aligned}
$$

Equations (9), (10), and (11) do not change whether the transverse shear deformation is considered or not. Also, the normal strains acting on the plane parallel to the middle surface are negligible compared to the other strain components. In other words, no stretching is present in the radial/normal direction, i.e. $\varepsilon_{r}=0$. The radial displacement is independent of thickness. $^{12}$

\subsection{First Order Shear Deformation Theory}

Denoting the rotations of the transverse plane as $\psi_{x}$ and $\psi_{\theta}$, the displacement field in a thin shell as well as in a thick shell with first order shear deformation theory is

$$
\begin{aligned}
& u_{x}=u_{x}^{0}+z \psi_{x} \\
& u_{\theta}=u_{\theta}^{0}+z \psi_{\theta}
\end{aligned}
$$

where $u_{x}^{0}$ and $u_{\theta}^{0}$ are the mid-surface displacements. In a thin shell, $\psi_{x}=-\frac{\partial u_{r}}{\partial x}$ and $\psi_{\theta}=-\frac{1}{a} \frac{\partial u_{r}}{\partial \theta}$. 
Donnell's first order shear deformation theory ${ }^{13}$ is used in the present formulation. Therefore, the transverse planes that are normal to the un-deformed mid-surface remain straight but not normal to the mid-surface after deformation. The rotations of the transverse planes are

$$
\begin{aligned}
& \psi_{x}=\gamma_{x r}-\frac{\partial u_{r}}{\partial x} \\
& \psi_{\theta}=\gamma_{\theta r}-\frac{1}{a} \frac{\partial u_{r}}{\partial \theta}
\end{aligned}
$$

In the first order shear deformation theory the curvatures are given by

$$
\begin{aligned}
\kappa_{x x} & =\frac{\partial \psi_{x}}{\partial x}=\frac{\partial \gamma_{x r}}{\partial x}-\frac{\partial^{2} u_{r}}{\partial x^{2}} \\
\kappa_{\theta \theta} & =\frac{1}{a} \frac{\partial \psi_{\theta}}{\partial \theta}=\frac{1}{a} \frac{\partial \gamma_{\theta r}}{\partial \theta}-\frac{1}{a^{2}} \frac{\partial^{2} u_{r}}{\partial \theta^{2}} \\
\kappa_{x \theta} & =\frac{\partial \psi_{\theta}}{\partial x}+\frac{1}{a} \frac{\partial \psi_{x}}{\partial \theta}=\frac{\partial \gamma_{\theta r}}{\partial x}-\frac{2}{a} \frac{\partial^{2} u_{r}}{\partial x \partial \theta}+\frac{1}{a} \frac{\partial \gamma_{x r}}{\partial \theta}
\end{aligned}
$$

Substituting the expressions for the rotations of the transverse plane [Eqs. (14-15)] and the displacement field [Eqs. (12-13)] in Eqs. (9-11), the strains become

$$
\begin{aligned}
\varepsilon_{x x} & =\varepsilon_{x x}^{0}+z \kappa_{x x} \\
\varepsilon_{\theta \theta} & =\varepsilon_{\theta \theta}^{0}+z \kappa_{\theta \theta} \\
\gamma_{x \theta} & =\gamma_{x \theta}^{0}+z \kappa_{x \theta}
\end{aligned}
$$

where $\varepsilon_{x x}^{0}, \varepsilon_{\theta \theta}^{0}$ and $\gamma_{x \theta}^{0}$ are the mid-surface strains.

The transverse shear strains will be constant across the section

$$
\begin{aligned}
& \gamma_{x r}=\gamma_{x r}^{0} \\
& \gamma_{\theta r}=\gamma_{\theta r}^{0}
\end{aligned}
$$

where $\gamma_{x r}^{0}$ and $\gamma_{\theta r}^{0}$ are the mid-surface shear strains

$$
\begin{aligned}
& \gamma_{x r}^{0}=\psi_{x}+\frac{\partial u_{r}}{\partial x} \\
& \gamma_{\theta r}^{0}=\psi_{\theta}+\frac{1}{a} \frac{\partial u_{r}}{\partial \theta} .
\end{aligned}
$$

It should be noted that the term $\frac{u_{\theta}}{a}$ is neglected in the expression for $\gamma_{\theta r}$ as per Donnell's theory, whereas other theories (Love's theory, Loo's theory, Sander's theory) include this term in the formulation. In this work Donnell's theory is used.

\subsection{Force and Moment Resultants}

The force and moment resultants are defined as in the case of thin shells as

$$
\begin{aligned}
& {\left[\begin{array}{l}
N_{x x} \\
N_{\theta \theta} \\
N_{x \theta}
\end{array}\right]=\sum_{k=1}^{k=n} \int_{h_{k-1}}^{h_{k}}\left[\begin{array}{l}
\sigma_{x x} \\
\sigma_{\theta \theta} \\
\tau_{x \theta}
\end{array}\right]_{k}\left[1+\frac{z}{R_{i}}\right] d z ;} \\
& {\left[\begin{array}{l}
M_{x x} \\
M_{\theta \theta} \\
M_{x \theta}
\end{array}\right]=\sum_{k=1}^{k=n} \int_{h_{k-1}}^{h_{k}}\left[\begin{array}{l}
\sigma_{x x} \\
\sigma_{\theta \theta} \\
\tau_{x \theta}
\end{array}\right]\left[1+\frac{z}{R_{i}}\right] z d z ;}
\end{aligned}
$$

where $R_{i}$ is the radii of curvature in the respective direction and $k$ refers to the number of layers in the laminate. For cylindrical shell, $R_{x}=\infty$ and $R_{\theta}=a$.
It is assumed that the term $\left[1+\frac{z}{R_{i}}\right]$ is very close to unity. ${ }^{15,16}$ The stresses are related to the strains through elastic stiffness coefficients denoted by $\overline{Q_{i j}}$, details of which are not given here for brevity but explained in Josephine Kelvina Florence and Ranji work. ${ }^{11}$ The strains are related to the midsurface strains and curvatures through Eqs. (19-21). Combining all the above relations the force and moment resultants become

$$
\left[\begin{array}{l}
N_{x x} \\
N_{\theta \theta} \\
N_{x \theta} \\
M_{x x} \\
M_{\theta \theta} \\
M_{x \theta}
\end{array}\right]=\left[\begin{array}{llllll}
A_{11} & A_{12} & A_{16} & B_{11} & B_{12} & B_{16} \\
A_{12} & A_{22} & A_{26} & B_{12} & B_{22} & B_{26} \\
A_{16} & A_{26} & A_{66} & B_{16} & B_{26} & B_{66} \\
B_{11} & B_{12} & B_{16} & D_{11} & D_{12} & D_{16} \\
B_{12} & B_{22} & B_{26} & D_{12} & D_{22} & D_{26} \\
B_{16} & B_{26} & B_{66} & D_{16} & D_{26} & D_{66}
\end{array}\right]\left[\begin{array}{c}
\varepsilon_{x x}^{0} \\
\varepsilon_{\theta \theta}^{0} \\
\gamma_{x \theta}^{0} \\
\kappa_{x x} \\
\kappa_{\theta \theta} \\
\kappa_{x \theta}
\end{array}\right]
$$

where $A_{i j}=\sum_{k=1}^{k=n}\left(\overline{Q_{i j}}\right)_{k}\left(h_{k}-h_{k-1}\right)$, also called as extensional stiffness terms; $B_{i j}=\frac{1}{2} \sum_{k=1}^{k=n}\left(\overline{Q_{i j}}\right)_{k}\left(h_{k}^{2}-h_{k-1}^{2}\right)$, also called as coupling stiffness terms; $D_{i j}=\frac{1}{3} \sum_{k=1}^{k=n}\left(\overline{Q_{i j}}\right)_{k}\left(h_{k}^{3}-\right.$ $\left.h_{k-1}^{3}\right)$, also called as bending stiffness terms; and $\overline{Q_{i j}}$ are coefficients of elastic stiffness.

In the present work the laminate considered is symmetric and balanced, therefore $B_{i j}=0 ; A_{16}=0 ; A_{26}=0$. Also, assume that $D_{16}$ and $D_{26}$ are negligible, the above relations become

$$
\left[\begin{array}{l}
N_{x x} \\
N_{\theta \theta} \\
N_{x \theta} \\
M_{x x} \\
M_{\theta \theta} \\
M_{x \theta}
\end{array}\right]=\left[\begin{array}{cccccc}
A_{11} & A_{12} & 0 & 0 & 0 & 0 \\
A_{12} & A_{22} & 0 & 0 & 0 & 0 \\
0 & 0 & A_{66} & 0 & 0 & 0 \\
0 & 0 & 0 & D_{11} & D_{12} & 0 \\
0 & 0 & 0 & D_{12} & D_{22} & 0 \\
0 & 0 & 0 & 0 & 0 & D_{66}
\end{array}\right]\left[\begin{array}{c}
\varepsilon_{x x}^{0} \\
\varepsilon_{\theta \theta}^{0} \\
\gamma_{x \theta}^{0} \\
\kappa_{x x} \\
\kappa_{\theta \theta} \\
\kappa_{x \theta}
\end{array}\right] .
$$

The above set of equations is the same as those for thin composite cylindrical shells, but the expressions for the curvatures include the transverse shear effects.

\subsection{Governing Differential Equations of Motion}

Using Airy stress function, the stress resultants can be defined as

$$
\begin{aligned}
& N_{x x}=\frac{1}{a^{2}} \frac{\partial^{2} \emptyset}{\partial \theta^{2}} \\
& N_{\theta \theta}=\frac{\partial^{2} \emptyset}{\partial x^{2}} \\
& N_{x \theta}=-\frac{1}{a^{2}} \frac{\partial^{2} \emptyset}{\partial x \partial \theta} .
\end{aligned}
$$

The function $\varnothing$ was first introduced by Airy and is in general known as Airy's stress function. ${ }^{17}$ Using this function, the first two equations of motion are satisfied completely. In other words, two independent in-plane displacements are eliminated and the unknowns reduce to two which are $\varnothing$ and $u_{r}$. For solving the two unknowns, we make use of the third equation of motion and an additional equation generated using compatibility condition.

Substituting Eq. (29), Eqs. (16-18) and Eq. (31) in Eq. (6), 
we get

$$
\begin{aligned}
& D_{11} \frac{\partial^{3} \gamma_{x r}}{\partial x^{3}}+ \frac{D_{22}}{a^{3}} \frac{\partial^{3} \gamma_{\theta r}}{\partial \theta^{3}}+ \\
& \frac{\left(D_{12}+2 D_{66}\right)}{a}\left\{\frac{\partial^{3} \gamma_{\theta r}}{\partial x^{2} \partial \theta}+\frac{1}{a} \frac{\partial^{3} \gamma_{x r}}{\partial x \partial \theta^{2}}\right\}= \\
& D_{11} \frac{\partial^{4} u_{r}}{\partial x^{4}}+\frac{2\left(D_{12}+2 D_{66}\right)}{a^{2}} \frac{\partial^{4} u_{r}}{\partial x^{2} \partial \theta^{2}}+ \\
& \frac{D_{22}}{a^{4}} \frac{\partial^{4} u_{r}}{\partial \theta^{4}}+\frac{1}{a} \frac{\partial^{2} \emptyset}{\partial x^{2}}+\rho_{m} \frac{\partial^{2} u_{r}}{\partial t^{2}}
\end{aligned}
$$

Equation (33) represents the first governing differential equation which is in terms of $\gamma_{x r}, \gamma_{\theta r}, u_{r}$ and $\emptyset$.

The terms involving $\gamma_{x r}, \gamma_{\theta r}$ need to be eliminated to have a closed form expression for the modal density. For this, we make use of force equilibrium consideration which is given below for ready reference

$$
\frac{\partial Q_{x r}}{\partial x}+\frac{1}{a} \frac{\partial Q_{\theta r}}{\partial \theta}-\frac{N_{\theta \theta}}{a}+q_{r}=0
$$

For a honeycomb sandwich construction, the average shear angle $\gamma_{x r}=\frac{Q_{x r}}{N_{x}}$ where $N_{x}=G_{x} h\left(1+\frac{t}{h}\right)^{2}$, ${ }^{18}$ where $G_{x}$ is the core shear modulus, $h$ is the thickness of core and $t$ is the thickness of face sheet. Assuming the core to be isotropic $G_{x}=G_{\theta}=G$ and $N_{x}=N_{\theta}=N$, called shear rigidity of the shell, the shear angle can be expressed as

$$
\gamma_{x r}=\frac{Q_{x r}}{N} ; \quad \gamma_{\theta r}=\frac{Q_{\theta r}}{N}
$$

Substituting Eq. (35) into Eq. (34), the force equilibrium equation becomes

$$
\frac{\partial \gamma_{x r}}{\partial x}+\frac{1}{a} \frac{\partial \gamma_{\theta r}}{\partial \theta}-\frac{1}{N a} \frac{\partial^{2} \varnothing}{\partial x^{2}}=-\frac{q_{r}}{N} .
$$

From Eq. (36), upon suitable algebraic operations, we get

$D_{11} \frac{\partial^{3} \gamma_{x r}}{\partial x^{3}}+\frac{D_{22}}{a^{3}} \frac{\partial^{3} \gamma_{\theta r}}{\partial \theta^{3}}=-\frac{1}{N}\left(D_{11} \frac{\partial^{2} q_{r}}{\partial x^{2}}+\frac{D_{22}}{a^{2}} \frac{\partial^{2} q_{r}}{\partial \theta^{2}}\right)+$ $\frac{1}{N}\left(\frac{D_{11}}{a} \frac{\partial^{4} \emptyset}{\partial x^{4}}+\frac{D_{22}}{a^{3}} \frac{\partial^{4} \varnothing}{\partial x^{2} \partial \theta^{2}}\right)-\frac{D_{11}}{a} \frac{\partial^{3} \gamma_{\theta r}}{\partial x^{2} \partial \theta}-\frac{D_{22}}{a^{2}} \frac{\partial^{3} \gamma_{x r}}{\partial x \partial \theta^{2}}$.

Substitution of Eq. (37) into Eq. (33) gives

$$
\begin{aligned}
& -\frac{1}{N}\left(D_{11} \frac{\partial^{2} q_{r}}{\partial x^{2}}+\frac{D_{22}}{a^{2}} \frac{\partial^{2} q_{r}}{\partial \theta^{2}}\right)+ \\
& \frac{1}{N}\left(\frac{D_{11}}{a} \frac{\partial^{4} \emptyset}{\partial x^{4}}+\frac{D_{22}}{a^{3}} \frac{\partial^{4} \emptyset}{\partial x^{2} \partial \theta^{2}}\right)+ \\
& \left(\frac{D_{12}+2 D_{66}-D_{11}}{a}\right) \frac{\partial^{3} \gamma_{\theta r}}{\partial \theta \partial x^{2}}+ \\
& \left(\frac{D_{12}+2 D_{66}-D_{22}}{a^{2}}\right) \frac{\partial^{3} \gamma_{x r}}{\partial x \partial \theta^{2}}=D_{11} \frac{\partial^{4} u_{r}}{\partial x^{4}}+ \\
& \frac{2\left(D_{12}+2 D_{66}\right)}{a^{2}} \frac{\partial^{4} u_{r}}{\partial x^{2} \partial \theta^{2}}+\frac{D_{22}}{a^{4}} \frac{\partial^{4} u_{r}}{\partial \theta^{4}}+\frac{1}{a} \frac{\partial^{2} \emptyset}{\partial x^{2}}+\rho_{m} \frac{\partial^{2} u_{r}}{\partial t^{2}} .
\end{aligned}
$$

It is not possible to eliminate $\gamma_{x r}$ and $\gamma_{\theta r}$ completely. If the terms having $\gamma_{x r}$ and $\gamma_{\theta r}$ are neglected, the differential equa- tion becomes

$-\frac{1}{N}\left(D_{11} \frac{\partial^{2} q_{r}}{\partial x^{2}}+\frac{D_{22}}{a^{2}} \frac{\partial^{2} q_{r}}{\partial \theta^{2}}\right)+$

$\frac{1}{N}\left(\frac{D_{11}}{a} \frac{\partial^{4} \emptyset}{\partial x^{4}}+\frac{D_{22}}{a^{3}} \frac{\partial^{4} \emptyset}{\partial x^{2} \partial \theta^{2}}\right)=D_{11} \frac{\partial^{4} u_{r}}{\partial x^{4}}+$

$\frac{2\left(D_{12}+2 D_{66}\right)}{a^{2}} \frac{\partial^{4} u_{r}}{\partial x^{2} \partial \theta^{2}}+\frac{D_{22}}{a^{4}} \frac{\partial^{4} u_{r}}{\partial \theta^{4}}+\frac{1}{a} \frac{\partial^{2} \emptyset}{\partial x^{2}}+\rho_{m} \frac{\partial^{2} u_{r}}{\partial t^{2}}$.

As $q_{r}=-\rho_{m} \frac{\partial^{2} u_{r}}{\partial t^{2}}$, the above differential equation becomes

$D_{11} \frac{\partial^{4} u_{r}}{\partial x^{4}}+\frac{2\left(D_{12}+2 D_{66}\right)}{a^{2}} \frac{\partial^{4} u_{r}}{\partial x^{2} \partial \theta^{2}}+\frac{D_{22}}{a^{4}} \frac{\partial^{4} u_{r}}{\partial \theta^{4}}+$

$\frac{1}{a} \frac{\partial^{2} \emptyset}{\partial x^{2}}+\rho_{m} \frac{\partial^{2} u_{r}}{\partial t^{2}}-\frac{\rho_{m}}{N} \frac{\partial^{2}}{\partial t^{2}}\left(D_{11} \frac{\partial^{2} u_{r}}{\partial x^{2}}+\frac{D_{22}}{a^{2}} \frac{\partial^{2} u_{r}}{\partial \theta^{2}}\right)-$

$\frac{1}{N}\left(\frac{D_{11}}{a} \frac{\partial^{4} \emptyset}{\partial x^{4}}+\frac{D_{22}}{a^{3}} \frac{\partial^{4} \emptyset}{\partial x^{2} \partial \theta^{2}}\right)=0$.

Equation (40) is the first governing differential equation in terms of Airy's stress function and normal displacements.

In this formulation, all the terms representing the shear effects are not included. However, if one considers the differential equation of a thin composite cylinder (which is given below for reference),

$$
\begin{aligned}
& D_{11} \frac{\partial^{4} u_{r}}{\partial x^{4}}+\frac{2\left(D_{12}+2 D_{66}\right)}{a^{2}} \frac{\partial^{4} u_{r}}{\partial x^{2} \partial \theta^{2}}+\frac{D_{22}}{a^{4}} \frac{\partial^{4} u_{r}}{\partial \theta^{4}}+ \\
& \frac{1}{a} \frac{\partial^{2} \emptyset}{\partial x^{2}}+\rho_{m} \frac{\partial^{2} u_{r}}{\partial t^{2}}=0 ;
\end{aligned}
$$

one can infer that most of the significant terms are included. It should also be noted that if the terms having $\gamma_{x r}$ and $\gamma_{\theta r}$ are not neglected, it will not be possible to incorporate shear effects, which will result in significant error. ${ }^{18}$ Since these terms are third derivatives of the shear angle it is expected that these are negligible.

To obtain the second differential equation, compatibility condition is enforced. This is carried out by eliminating the displacements from the strain displacement relationships and is given by Eq. (42) as

$$
\frac{\kappa_{x x}}{a}+\frac{\partial^{2} \varepsilon_{\theta \theta}^{0}}{\partial x^{2}}-\frac{1}{a} \frac{\partial^{2} \gamma_{x \theta}^{0}}{\partial x \partial \theta}+\frac{1}{a^{2}} \frac{\partial^{2} \varepsilon_{x x}^{0}}{\partial \theta^{2}}-\frac{1}{a} \frac{\partial \gamma_{x r}}{\partial x}=0 .
$$

Using Eq. (29) and the definition of Airy stress function as given in Eqs. (31-32), the mid-surface strains can be written as

$$
\left[\begin{array}{c}
\varepsilon_{x x}^{0} \\
\varepsilon_{\theta \theta}^{0} \\
\gamma_{x \theta}^{0}
\end{array}\right]=\frac{1}{A_{11} A_{22}-A_{12}^{2}}\left[\begin{array}{ccc}
A_{22} & -A_{12} & 0 \\
-A_{12} & A_{11} & 0 \\
0 & 0 & \frac{1}{A_{66}}
\end{array}\right]\left[\begin{array}{c}
\frac{1}{a^{2}} \frac{\partial^{2} \emptyset}{\partial \theta^{2}} \\
\frac{\partial^{2} \emptyset}{\partial x^{2}} \\
-\frac{1}{a^{2}} \frac{\partial^{2} \emptyset}{\partial x \partial \theta}
\end{array}\right]
$$

Using Eq. (31) and Eq. (43) in Eq. (42), we get

$$
\begin{aligned}
& \frac{A_{12}^{2}-A_{11} A_{22}}{a} \frac{\partial^{2} u_{r}}{\partial x^{2}}+A_{11} \frac{\partial^{4} \emptyset}{\partial x^{4}}+ \\
& \frac{1}{a^{2}} \frac{A_{11} A_{22}-A_{12}^{2}-2 A_{11} A_{66}}{A_{66}} \frac{\partial^{4} \emptyset}{\partial x^{2} \partial \theta^{2}}+\frac{A_{22}}{a^{4}} \frac{\partial^{4} \emptyset}{\partial \theta^{4}}=0 .
\end{aligned}
$$

This is the second equation in terms of $\varnothing$ and $u_{r}$.

Hence, Eqs. (40) and (44) are the governing differential equations in terms of Airy's stress function and normal displacements. 


\subsection{Assumptions}

Though the assumptions involved in arriving at the differential equations are presented whenever they are applied they are summarized here to have an overall idea. Some of these assumptions are part of any two-dimensional structural analysis but given here for completeness.

1. The shell is cylindrical.

2. The shell is shallow; the term $\left[1+\frac{z}{R_{i}}\right]$ is very close to one. The radius of curvature is very large compared to the in-plane displacements.

3. Material is linearly elastic.

4. The laminate is symmetric, therefore $B_{i j}=0$.

5. The laminate is balanced, therefore $A_{16}, A_{26}=0$.

6. $D_{16}$ and $D_{26}$ are negligible.

7. Plane stress condition exists.

8. $\varepsilon_{r}=0$, i.e., the displacement $u_{r}$ is independent of $z$.

9. The transverse shear strains in the face sheets are neglected.

10. Mass distribution is uniform, i.e. mass per unit area is constant.

11. Rotary inertia is neglected.

12. In a honeycomb sandwich construction the core is homogeneous; the wavelength is far greater than the cell size.

13. The transverse planes that are normal to the un-deformed layers deform. The transverse deflection is due to both shear force and bending moment.

14. The transverse plane remains straight but not normal to the mid surface after deformation. Mindlin's theory is used. The rotation of the transverse plane $\left(\gamma_{x r}\right.$ and $\left.\gamma_{\theta r}\right)$ is independent of the thickness of the shell and for this the average value of the shear angle is used.

15. Donnell's shear deformable theory is adopted. Influence of inertia force in the in-plane direction is neglected. This is restricted to normal loading.

16. The displacements $u_{x}$ and $u_{\theta}$ are not independent but related by Airy's stress function.

\section{NATURAL FREQUENCY}

To determine the modal density and mode count, an expression for natural frequency needs to be available. As no closed form expression for natural frequency of composite cylindrical shell considering transverse shear deformation has been reported, derivation of the same is first carried out. Solution to differential equations for free vibration with simply supported boundary conditions along the curved edges of the cylinder lead to the required expression for natural frequency. The boundary conditions are $u_{r}(0, \theta, t)=u_{r}(L, \theta, t)=0$, $M(0, \theta, t)=M(L, \theta, t)=0$. These boundary conditions are satisfied by

$$
\begin{aligned}
& u_{r}(x, \theta, t)=U_{m n} \sin \frac{m \pi x}{L} \cos n(\theta-\delta) e^{j \omega t} \\
& \varnothing(x, \theta, t)=\emptyset_{m n} \sin \frac{m \pi x}{L} \cos n(\theta-\delta) e^{j \omega t}
\end{aligned}
$$

for $m$ half waves in the axial direction and $n$ full waves in the circumferential direction. The parameter $\delta$ represents an arbitrary angle indicating that there is no preference in circumferential direction.

Upon substitution of Eqs. (45) and (46) in the governing differential equations we get

$$
\left|\begin{array}{ll}
M_{11} & M_{12} \\
M_{21} & M_{22}
\end{array}\right| \times\left\{\begin{array}{l}
U_{m n} \\
\emptyset_{m n}
\end{array}\right\}=0 ;
$$

where

$$
\begin{aligned}
M_{11}= & D_{11}\left(\frac{m \pi}{L}\right)^{4}+2\left(D_{12}+2 D_{66}\right)\left(\frac{m \pi}{L}\right)^{2}\left(\frac{n}{a}\right)^{2}+ \\
& D_{22}\left(\frac{n}{a}\right)^{4}-\rho_{m} \omega^{2}-\frac{\rho_{m} \omega^{2}}{N}\left(D_{11}\left(\frac{m \pi}{L}\right)^{2}+D_{22}\left(\frac{n}{a}\right)^{2}\right) ; \\
M_{12}= & -\left\{\frac{1}{a}\left(\frac{m \pi}{L}\right)^{2}+\frac{1}{a N}\left(D_{11}\left(\frac{m \pi}{L}\right)^{4}+D_{22}\left(\frac{m \pi}{L}\right)^{2}\left(\frac{n}{a}\right)^{2}\right)\right\} ; \\
M_{21}= & \frac{A_{11} A_{22}-A_{12}^{2}}{a}\left(\frac{m \pi}{L}\right)^{2} ; \\
M_{22}= & A_{11}\left(\frac{m \pi}{L}\right)^{4}+A_{22}\left(\frac{n}{a}\right)^{4}+ \\
& \frac{A_{11} A_{22}-A_{12}^{2}-2 A_{12} A_{66}}{A_{66}}\left(\frac{m \pi}{L}\right)^{2}\left(\frac{n}{a}\right)^{2} .
\end{aligned}
$$

Setting the determinant of the above matrix to zero gives the expression for natural frequency as Eq. (48). Equation (48) is the expression for natural frequency of orthotropic cylindrical shells considering transverse shear deformation of first order.

If transverse shear effects are neglected, i.e. when $N$ tends to $\infty$, the expression for the natural frequency becomes Eq. (49), which is the same as that reported for thin composite cylinders. ${ }^{11}$

To assess the influence of transverse shear on the natural frequencies of typical honeycomb sandwich composite cylinder used in spacecraft the natural modes are obtained considering the transverse shear [Eq. (48)] as well as neglecting the transverse shear [Eq. (49)]. The length of the cylinder is $3000 \mathrm{~mm}$ and the mean radius is $600 \mathrm{~mm}$.

Material properties of the sandwich cylinder are given below.

\section{Face sheet}

Material: 2 layers of bidirectional CFRP.

Each layer has the following properties:

Young's modulus $=1.47 \times 10^{11} \mathrm{~N} / \mathrm{m}^{2}$

Shear modulus $=4 \times 10^{9} \mathrm{~N} / \mathrm{m}^{2}$

Poisson's ratio $=0.03$

Thickness $=0.08 \mathrm{~mm}$

Density $=1660 \mathrm{~kg} / \mathrm{m}^{3}$

\section{Core}

Material: Aluminium honey comb

Thickness $=12 \mathrm{~mm}$

Density $=32 \mathrm{~kg} / \mathrm{m}^{3}$

Shear modulus $=1.4 \times 10^{8} \mathrm{~N} / \mathrm{m}^{2}$

The cross-section of the cylinder has the following elastic properties: $A_{11}=4.71 \times 10^{7} \mathrm{~N} / \mathrm{m}, A_{22}=4.71 \times 10^{7} \mathrm{~N} / \mathrm{m}$, $A_{12}=1.41 \times 10^{6} \mathrm{~N} / \mathrm{m}, A_{66}=1.28 \times 10^{6} \mathrm{~N} / \mathrm{m}, D_{11}=1.74 \times$ $10^{3} \mathrm{Nm}, D_{22}=1.74 \times 10^{3} \mathrm{Nm}, D_{12}=52.2 \mathrm{Nm}$, and $D_{66}=$ $47.3 \mathrm{Nm}$. Shear rigidity of the section is $17.25 \times 10^{5} \mathrm{~N} / \mathrm{m}$. Mass per unit area of the cylinder is $0.92 \mathrm{~kg} / \mathrm{m}^{2}$ (includes 2 face sheets, core and adhesive).

As the transverse shear effects are expected to be significant for higher order modes the results are given in Table 1 for the higher order modes. The results show the need for the inclusion of transverse shear deformation even for these cylinders.

\section{MODE COUNT AND MODAL DENSITY}

Modal density is the average number of modes per unit frequency. Modal density can be determined from the constant 


$$
\begin{aligned}
& \omega_{m n}^{2}=\frac{1}{\rho_{m}\left[1+\left(\frac{D_{11}}{N}\left(\frac{m \pi}{L}\right)^{2}+\frac{D_{22}}{N}\left(\frac{n}{a}\right)^{2}\right)\right]}\left\{D_{11}\left(\frac{m \pi}{L}\right)^{4}+2\left(D_{12}+2 D_{66}\right)\left(\frac{m \pi}{L}\right)^{2}\left(\frac{n}{a}\right)^{2}+D_{22}\left(\frac{n}{a}\right)^{4}+\right. \\
& \left.\frac{\frac{A_{11} A_{22}-A_{12}^{2}}{a}\left(\frac{m \pi}{L}\right)^{2} \times\left\{\frac{1}{a}\left(\frac{m \pi}{L}\right)^{2}+\frac{1}{a N}\left(D_{11}\left(\frac{m \pi}{L}\right)^{4}+D_{22}\left(\frac{m \pi}{L}\right)^{2}\left(\frac{n}{a}\right)^{2}\right)\right\}}{A_{11}\left(\frac{m \pi}{L}\right)^{4}+A_{22}\left(\frac{n}{a}\right)^{4}+\frac{A_{11} A_{22}-A_{12}^{2}-2 A_{12} A_{66}}{A_{66}}\left(\frac{m \pi}{L}\right)^{2}\left(\frac{n}{a}\right)^{2}}\right\} \\
& \omega_{m n}^{2}=\frac{1}{\rho_{m}}\left\{D_{11}\left(\frac{m \pi}{L}\right)^{4}+2\left(D_{12}+2 D_{66}\right)\left(\frac{m \pi}{L}\right)^{2}\left(\frac{n}{a}\right)^{2}+D_{22}\left(\frac{n}{a}\right)^{4}+\right. \\
& \left.\frac{\left(\frac{m \pi}{L}\right)^{4} \frac{A_{11} A_{22}-A_{12}^{2}}{a^{2}}}{A_{11}\left(\frac{m \pi}{L}\right)^{4}+A_{22}\left(\frac{n}{a}\right)^{4}+\frac{A_{11} A_{22}-A_{12}^{2}-2 A_{12} A_{66}}{A_{66}}\left(\frac{m \pi}{L}\right)^{2}\left(\frac{n}{a}\right)^{2}}\right\} \text {. }
\end{aligned}
$$

Table 1. Comparison of natural frequencies of a cylinder with and without shear deformation.

\begin{tabular}{|c|c|c|c|}
\hline $\begin{array}{c}m, \\
\text { axial half } \\
\text { wave } \\
\text { number }\end{array}$ & $\begin{array}{c}n, \\
\text { circumferential } \\
\text { full wave } \\
\text { number }\end{array}$ & $\begin{array}{c}\text { Frequency } \\
\text { neglecting } \\
\text { shear } \\
\text { deformation }(\mathrm{Hz})\end{array}$ & $\begin{array}{c}\text { Frequency } \\
\text { considering } \\
\text { shear } \\
\text { deformation }(\mathrm{Hz})\end{array}$ \\
\hline 9 & 2 & 1019 & 1002 \\
10 & 3 & 1008 & 970 \\
6 & 7 & 1018 & 941 \\
7 & 7 & 1062 & 975 \\
10 & 12 & 2944 & 2394 \\
20 & 11 & 4004 & 3008 \\
15 & 17 & 5967 & 4161 \\
16 & 18 & 6700 & 4528 \\
30 & 15 & 8420 & 5204 \\
28 & 20 & 10144 & 5872 \\
\hline
\end{tabular}

' $\omega$ ' curve in the wave number plane. The number of modal points enclosed by the curve gives the number of modes below the frequency $\omega$. The derivative of the expression for the number of modes below the frequency $\omega$, with respect to the frequency gives modal density.

\subsection{Expression for Mode Count}

Mode count, denoted by $N(\omega)$, is the number of modes below the frequency $\omega$ and it can be obtained from the ratio of the area enclosed by the constant $\omega$ curve to the area that corresponds to one mode. Mathematically, mode count for two dimensional surfaces in the wave domain can be written as

$$
N(\omega)=\frac{A}{\pi^{2}} \oiint d K_{1} d K_{2}
$$

where $K_{1}$ and $K_{2}$ are the wave numbers in the principal directions of the cylindrical shell surface and $A$ corresponds to the area of the cylinder.

$$
N(\omega)=\frac{a L}{\pi} \int_{0}^{\frac{\pi}{2}} r^{2} d \theta
$$

By defining the wave numbers as $K_{1}=\frac{m \pi}{L}$ and $K_{2}=\frac{n}{a}$ in Eq. (48) and using a polar coordinate system in the wave space, the expression for $r^{2}$ can be obtained as

$$
r^{4} \frac{f_{1}}{\rho_{m} \omega_{m n}^{2}-f_{3}}-\frac{r^{2} f_{2}}{N}-1=0 ;
$$

where $f_{1}, f_{2}$, and $f_{3}$ are functions of $\theta$ (given below) representing the orthotropic elastic properties:
$f_{1}=D_{11} c^{4}+2\left(D_{12}+2 D_{66}\right) c^{2} s^{2}+D_{22} s^{4} ;$

$f_{2}=D_{11} c^{2}+D_{22} s^{2}$;

$f_{3}=\frac{\frac{\left(A_{11} A_{22}-A_{12}^{2}\right) c^{4}}{a^{2}}}{A_{11} c^{4}+A_{22} s^{4}+\frac{A_{11} A_{22}-A_{12}^{2}-2 A_{12} A_{66}}{A_{66}} c^{2} s^{2}}$.

Solving for $r^{2}$, we get

$$
r^{2}=\frac{\rho_{m} \omega_{m n}^{2}-f_{3}}{2 f_{1}}\left\{\frac{f_{2}}{N} \pm \sqrt{\left(\frac{f_{2}}{N}\right)^{2}+\frac{4 f_{1}}{\rho_{m} \omega_{m n}^{2}-f_{3}}}\right\} .
$$

Considering the positive root of $r^{2}$ and using it in Eq. (50) and changing $\omega_{m n}$ to $\omega$, we get the expression for the mode count as

$$
N(\omega)=\frac{a L}{\pi} \int_{0}^{\frac{\pi}{2}}\left[\frac{\rho_{m} \omega^{2}-f_{3}}{2 f_{1}}\left\{\frac{f_{2}}{N}+\sqrt{\left(\frac{f_{2}}{N}\right)^{2}+\frac{4 f_{1}}{\rho_{m} \omega_{m n}^{2}-f_{3}}}\right\}\right] d \theta .
$$

Equation (53) is the expression for mode count of sandwich cylindrical shells considering transverse shear deformation.

\subsection{Expression for Modal Density}

Modal density, denoted by $n(\omega)$, is related to mode count by the relation $n(\omega)=\frac{d N(\omega)}{d \omega}$. It is to be noted that modal density as a function of frequency is expressed as $n(f)=2 \pi n(\omega)$.

Differentiating the expression for mode count given by Eq. (53) with respect to ' $\omega$ ', we get

$$
\begin{gathered}
n(\omega)=\frac{\rho_{m} \omega a L}{N \pi} \int_{0}^{\frac{\pi}{2}}\left\{\left(\frac{f_{2}}{f_{1}}+\sqrt{\left(\frac{f_{2}}{f_{1}}\right)^{2}+\frac{4 N^{2}}{f_{1}\left(\rho_{m} \omega^{2}-f_{3}\right)}}\right)-\right. \\
\left.f_{1}\left(\rho_{m} \omega^{2}-f_{3}\right) \sqrt{\left(\frac{f_{2}}{f_{1}}\right)^{2}+\frac{4 N^{2}}{f_{1}\left(\rho_{m} \omega^{2}-f_{3}\right)}}\right\} d \theta ; \\
n(f)=\frac{2 A f \rho_{m}}{N} \int_{0}^{\frac{\pi}{2}}\left\{\left(\frac{f_{2}}{f_{1}}+\sqrt{\left(\frac{f_{2}}{f_{1}}\right)^{2}+\frac{4 N^{2}}{f_{1}\left(\rho_{m} \omega^{2}-f_{3}\right)}}\right)-\right. \\
\left.f_{1}\left(\rho_{m} \omega^{2}-f_{3}\right) \sqrt{\left(\frac{f_{2}}{f_{1}}\right)^{2}+\frac{4 N^{2}}{f_{1}\left(\rho_{m} \omega^{2}-f_{3}\right)}}\right\} d \theta .
\end{gathered}
$$




\begin{tabular}{l}
\hline \hline Table 2. Modal density using derived expression and finite element method. \\
\begin{tabular}{|c|c|c||}
\hline $\begin{array}{c}\text { Frequency } \\
\text { (Hz) }\end{array}$ & Expression from Eq. (55) & Finite element method \\
\hline 500 & 0.132 & 0.139 \\
630 & 0.161 & 0.137 \\
800 & 0.210 & 0.211 \\
1000 & 0.263 & 0.272 \\
1250 & 0.246 & 0.271 \\
1600 & 0.261 & 0.270 \\
2000 & 0.290 & 0.330 \\
2500 & 0.332 & 0.377 \\
\hline
\end{tabular}
\end{tabular}

Equation (55) gives the modal density for a composite cylindrical shell at frequency $f$, having a surface area of $A$ and considering the transverse shear deformation.

\section{COMPARISON WITH THE RESULTS OF FINITE ELEMENT METHOD}

Modal densities for a typical honeycomb sandwich cylinder with composite face sheets are obtained using the expression derived and they are compared with those obtained using the finite element method. The length of the cylinder considered is $2260 \mathrm{~mm}$, mean radius is $452 \mathrm{~mm}$, which results in a surface area of $6.42 \mathrm{~m}^{2}$.

Material properties of the above sandwich cylinder are given below.

Face sheet

Material: 4 layers (0/-35/0/35) of CFRP [1 layer of Bidirectional CFRP + 3 layers of Unidirectional CFRP]

Thickness $=0.365 \mathrm{~mm}$

Young's modulus of Unidirectional lamina

$$
=2.15 \times 10^{11} \mathrm{~N} / \mathrm{m}^{2} \text { (along the fibre direction) }
$$

$=6.6 \times 10^{9} \mathrm{~N} / \mathrm{m}^{2}$ (along the transverse direction)

Shear modulus of Unidirectional lamina $=3.9 \times 10^{9} \mathrm{~N} / \mathrm{m}^{2}$

Major Poisson's ratio $=0.23$

Density $=1600 \mathrm{~kg} / \mathrm{m}^{3}$

Properties of the Bidirectional lamina are the same as those described in Section 3.

Core

Material: Aluminium honeycomb

Thickness $=12 \mathrm{~mm}$

Shear modulus $=1.4 \times 10^{8} \mathrm{~N} / \mathrm{m}^{2}$

Density $=32 \mathrm{~kg} / \mathrm{m}^{3}$

The cross-section of the cylinder has the following elastic properties: $A_{11}=1.03 \times 10^{8} \mathrm{~N} / \mathrm{m}, A_{22}=0.363 \times 10^{8} \mathrm{~N} / \mathrm{m}$, $A_{12}=0.186 \times 10^{8} \mathrm{~N} / \mathrm{m}, A_{66}=1.99 \times 10^{7} \mathrm{~N} / \mathrm{m}, D_{11}=$ $3.95 \times 10^{3} \mathrm{Nm}, D_{22}=1.43 \times 10^{3} \mathrm{Nm}, D_{12}=0.705 \times 10^{3} \mathrm{Nm}$, and $D_{66}=0.753 \times 10^{3} \mathrm{Nm}$. The other elastic properties are $D_{16}=0.03 \times 10^{3} \mathrm{Nm}, D_{26}=0.015 \times 10^{3} \mathrm{Nm}$. Shear rigidity of the section is $17.83 \times 10^{5} \mathrm{~N} / \mathrm{m}$. Mass per unit area of the cylinder is $4.7 \mathrm{~kg} / \mathrm{m}^{2}$ (includes 2 face sheets, core and adhesive).

Modal densities computed using the expression given by Eq. (55) are given in Table 2 and Fig. 2. Finite element model is developed for the cylindrical geometry using 4-noded quadrilateral element. It is a shell element with 5 degreesof-freedom for each node. The face sheets and the core together are considered as a laminate. The elements are assigned with the properties of the laminate. The elastic properties of the laminate are determined from the properties of each layer. To represent transverse shear deformation, Mindlin's theory is used and the shear effects are included in the finite element model in terms of shear correction factor.

Natural frequencies of the normal modes of this cylinder are determined using NASTRAN solver. All the modes up to

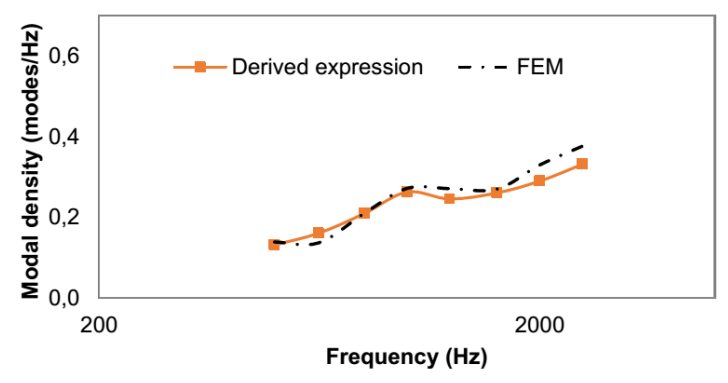

Figure 2. Comparison of modal densities using expression from Eq. (55) and finite element method.

$3000 \mathrm{~Hz}$ are extracted. Modal density for each one third octave band is then computed as the ratio of the number of modes in that band to the bandwidth. The results are given in Fig. 2 and a very good agreement is seen validating the expression derived.

\section{EXPRESSION FOR MODAL DENSITY IN SPECIAL CASES}

Modal density of a composite cylindrical shell considering transverse shear deformation can be obtained using Eq. (55). It is essential to verify whether the derived expression under limiting cases converges to the existing expressions. Also, the expression can be in a simple form under certain conditions. These are discussed here.

\subsection{Negligible Transverse Shear Deformations}

When the transverse shear effects are negligible, expression for modal density of sandwich cylinder should converge to that of thin composite cylinders. ${ }^{11}$ This condition can be achieved by taking $N$ to $\infty$. Taking $N$ inside the braces of general expression, we get

$$
\begin{gathered}
n(f)=2 A f \rho_{m} \int_{0}^{\frac{\pi}{2}}\left\{\left(\frac{f_{2}}{N f_{1}}+\sqrt{\left(\frac{f_{2}}{N f_{1}}\right)^{2}+\frac{4}{f_{1}\left(\rho_{m} \omega^{2}-f_{3}\right)}}\right)-\right. \\
\left.f_{1}\left(\rho_{m} \omega^{2}-f_{3}\right) \sqrt{\left(\frac{f_{2}}{N f_{1}}\right)^{2}+\frac{4}{f_{1}\left(\rho_{m} \omega^{2}-f_{3}\right)}}\right\} d \theta
\end{gathered}
$$

As $N$ tends to $\infty$,

$$
\begin{gathered}
n(f)=2 A f \rho_{m} \int_{0}^{\frac{\pi}{2}}\left\{\left(\sqrt{\frac{4}{f_{1}\left(\rho_{m} \omega^{2}-f_{3}\right)}}\right)-\right. \\
\left.\frac{2}{f_{1}\left(\rho_{m} \omega^{2}-f_{3}\right) \sqrt{\frac{4}{f_{1}\left(\rho_{m} \omega^{2}-f_{3}\right)}}}\right\} d \theta .
\end{gathered}
$$

On simplification

$$
n(f)=2 A f \sqrt{\rho_{m}} \int_{0}^{\frac{\pi}{2}} \frac{d \theta}{\sqrt{f_{1}} \sqrt{\omega^{2}-\frac{f_{3}}{\rho_{m}}}} .
$$

Upon substitution of $f_{1}$ and $f_{3}$, we get Eq. (59), which is the same as the expression reported for modal density of thin composite cylindrical shells. ${ }^{11}$ When the shear effects are neglected, the expression for modal density derived here converges to that of thin composite cylindrical shells. 


$$
n(f)=\frac{A}{\pi} \sqrt{\rho_{m}} \int_{0}^{\frac{\pi}{2}} \frac{d \theta}{\left\{1-\frac{c^{4}\left(A_{11} A_{22}-A_{12}^{2}\right)}{4 \pi^{2} f^{2} \rho_{m} a^{2}\left(A_{11} c^{4}+A_{22} s^{4}+\frac{A_{11} A_{22}-A_{12}^{2}-2 A_{12} A_{66}}{A_{66}} c^{2} s^{2}\right)}\right\}^{\frac{1}{2}}\left\{D_{11} c^{4}+2\left(D_{12}+2 D_{66}\right) c^{2} s^{2}+D_{22} s^{4}\right\}^{\frac{1}{2}}} .
$$

\subsection{Isotropic Shells}

For a cylinder with isotropic material, $A_{11}=A_{22}, D_{11}=$ $D_{22}=D$. The functions of $\theta$ become

$f_{1}=D_{11} c^{4}+2\left(D_{12}+2 D_{66}\right) c^{2} s^{2}+D_{22} s^{4}=$ $D\left(1-2 c^{2} s^{2}+\gamma c^{2} s^{2}\right)$;

$f_{2}=D_{11} c^{2}+D_{22} s^{2}=D_{11}=D$;

$f_{3}=\frac{A_{11} c^{4}\left(1-\alpha^{2}\right)}{a^{2}\left\{1-2 c^{2} s^{2}+\frac{1-\alpha^{2}-2 \alpha \beta}{\beta} c^{2} s^{2}\right\}}$;

where $\frac{A_{12}}{A_{11}}=\alpha ; \frac{A_{66}}{A_{11}}=\beta ; \frac{2\left(D_{12}+2 D_{66}\right)}{D_{11}}=\gamma$. For an isotropic cylinder, $\alpha=\mu ; \beta=\frac{1-\mu}{2} ; \gamma=2$.

The product $\cos ^{2} \theta \sin ^{2} \theta$ is approximately constant except for values of $\theta$ near 0 and $\pi / 2$. Since there is no preference for a wave number component, in other words equal probability of occurrence for the wave components, an average value of $\cos ^{2} \theta \sin ^{2} \theta$ is proposed to be used which is equal to $0.125 .{ }^{11}$ It should be noted that this approximation holds good as long as $\beta>0.2$ and for an isotropic cylinder $\beta$ is 0.35 . Making use of this approximation we get $f_{1}=D ; f_{2}=D$; $f_{3}=\frac{A_{11} c^{4}\left(1-\mu^{2}\right)}{a^{2}}$. Upon substitution of these functions the expression for modal density becomes

$$
\begin{aligned}
& n(f)=\frac{2 A f \rho_{m}}{N} \int_{0}^{\frac{\pi}{2}}\left\{\left(1+\sqrt{1+\frac{4 N^{2}}{D\left(\rho_{m} \omega^{2}-\frac{A_{11} c^{4}\left(1-\mu^{2}\right)}{a^{2}}\right)}}\right)-\right. \\
& \left.\frac{2 N^{2}}{D\left(\rho_{m} \omega^{2}-\frac{A_{11} c^{4}\left(1-\mu^{2}\right)}{a^{2}}\right) \sqrt{1+\frac{4 N^{2}}{D\left(\rho_{m} \omega^{2}-\frac{A_{11} c^{4}\left(1-\mu^{2}\right)}{a^{2}}\right)}}}\right\} d \theta .
\end{aligned}
$$

Defining $f(\theta)=D\left(\rho_{m} \omega^{2}-\frac{A_{11} c^{4}\left(1-\mu^{2}\right)}{a^{2}}\right)$, the above expression reduces to

$n(f)=\frac{2 A f \rho_{m}}{N} \int_{0}^{\frac{\pi}{2}}\left\{1+\sqrt{1+\frac{4 N^{2}}{f(\theta)}}-\frac{2 N^{2}}{\sqrt{\left(f(\theta)+4 N^{2}\right) f(\theta)}}\right\} d \theta$.

Equation (61) gives the expression for modal density of isotropic cylinders considering transverse shear deformation.

It will be interesting to compare the results given by Eq. (61) and those obtained by Wilkinson's expression ${ }^{4}$ for a typical sandwich cylinder with isotropic face sheets. The cylinder considered has a length of $3000 \mathrm{~mm}$, mean radius of $600 \mathrm{~mm}$ resulting in an area of $11.3 \mathrm{~m}^{2}$. The honeycomb core has a density of $32 \mathrm{~kg} / \mathrm{m}^{3}$ and the thickness of the core is $12 \mathrm{~mm}$. The face sheet has a thickness of $0.3 \mathrm{~mm}$ made of Aluminium. Young's modulus of Aluminium is considered as $7.2 \times 10^{10} \mathrm{~N} / \mathrm{m}^{2}$. The mass per unit area of the cylinder is $0.9151 \mathrm{~kg} / \mathrm{m}^{2}$.

The modal densities of the cylinder whose properties are given above are computed using Eq. (61) and compared with the results obtained using the expression derived by Wilkinson $^{4}$ in Fig. 3. The results show that the modal densities deter-

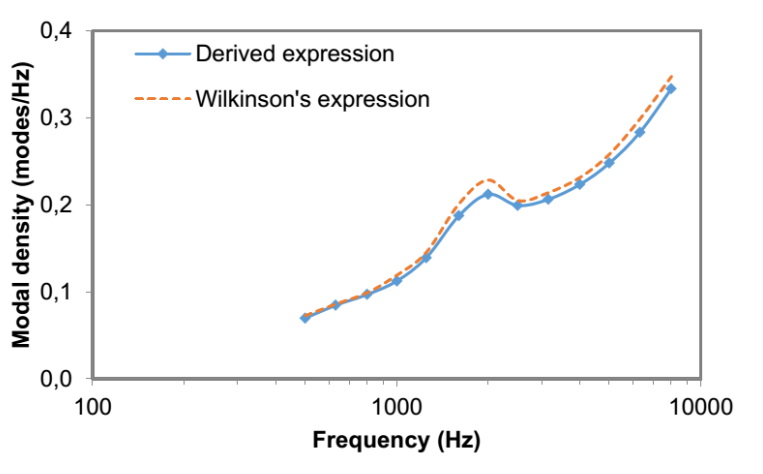

Figure 3. Modal densities of sandwich cylinders with isotropic face sheets.

mined using the expression derived here converges to those by the Wilkinson's expression when the cylinder is isotropic.

\subsection{Modal Density at High Frequencies}

At very high frequencies, the structural wavelengths are very much lower compared to the circumference so that a cylinder tends to behave like a flat plate. ${ }^{6}$ The expression at high frequencies can be obtained by setting $R$ to $\infty$ as

$$
\begin{aligned}
n(f)= & \frac{2 A f \rho_{m}}{N} \int_{0}^{\frac{\pi}{2}}\left\{\left(\frac{f_{2}}{f_{1}}+\sqrt{\left(\frac{f_{2}}{f_{1}}\right)^{2}+\frac{4 N^{2}}{\rho_{m} \omega^{2} f_{1}}}\right)-\right. \\
& \left.\frac{2 N^{2}}{\rho_{m} \omega^{2} f_{1} \sqrt{\left(\frac{f_{2}}{f_{1}}\right)^{2}+\frac{4 N^{2}}{\rho_{m} \omega^{2} f_{1}}}}\right\} d \theta
\end{aligned}
$$

where $f_{1}=D_{11} c^{4}+2\left(D_{12}+2 D_{66}\right) c^{2} s^{2}+D_{22} s^{4}$; $f_{2}=D_{11} c^{2}+D_{22} s^{2}$.

It is interesting to compare the modal densities obtained using Eq. (62) with those using the expression for the composite flat panels, considering transverse shear deformation, reported by Renji ${ }^{18}$ which is given as Eq. (63)

$$
\begin{aligned}
n(f)= & \frac{2 a b \rho f}{N} \int_{0}^{\frac{\pi}{2}}\left\{\frac{f_{2}}{f_{1}}+\frac{1}{f_{1}}\left(\rho^{2} \omega^{4} f_{2}^{2}+\frac{4 \rho \omega^{2} N^{2}}{\sqrt{D_{11} D_{22}}}\right)^{-\frac{1}{2}}\right. \\
& \left.\left(\rho \omega^{2} f_{2}^{2}+\frac{2 N^{2} f_{1}}{\sqrt{D_{11} D_{22}}}\right)\right\} d \theta ;
\end{aligned}
$$

where $f_{1}=1-\gamma_{1}^{2} \sin ^{2} 2 \theta$ with $\gamma_{1}^{2}=\frac{1}{2}\left\{1-\frac{D_{12}+2 D_{66}}{\sqrt{D_{11} D_{22}}}\right\}$; $f_{2}=\left(\frac{D_{11}}{D_{22}}\right)^{\frac{1}{4}} c^{2}+\left(\frac{D_{22}}{D_{11}}\right)^{\frac{1}{4}} s^{2}$.

It should be noted that the two equations are similar in nature. The forms, in which the wave numbers $\left(K_{1}\right.$ and $\left.K_{2}\right)$ and the functions of $\theta\left(f_{1}\right.$ and $\left.f_{2}\right)$ are defined, are different in both the expressions. In the case of shells, wave numbers are defined as $K_{1}=r \cos \theta ; K_{2}=r \sin \theta$, whereas they are defined 


Table 3. Modal densities of a honeycomb sandwich composite cylinder.
\begin{tabular}{||c|c|c||}
\hline $\begin{array}{c}\text { Frequency } \\
\text { (Hz) }\end{array}$ & With transverse shear & Wodal density (modes/Hz) \\
\hline 500 & 0.132 & 0.139 \\
630 & 0.161 & 0.137 \\
800 & 0.210 & 0.211 \\
1000 & 0.263 & 0.272 \\
1250 & 0.246 & 0.271 \\
1600 & 0.261 & 0.270 \\
2000 & 0.290 & 0.330 \\
2500 & 0.332 & 0.377 \\
3150 & 0.390 & 0.147 \\
4000 & 0.473 & 0.144 \\
5000 & 0.575 & 0.145 \\
6300 & 0.712 & 0.145 \\
8000 & 0.895 & 0.145 \\
\hline
\end{tabular}

as $D_{11}^{\frac{1}{4}} K_{1}=r \cos \theta ; D_{22}^{\frac{1}{4}} K_{2}=r \sin \theta$ for plates. As an example, both the expressions give a modal density of 0.5 modes $/ \mathrm{Hz}$ at $10000 \mathrm{~Hz}$ for the cylinder described in Section 3.

\subsection{Modal Density for Cylinders Having Equal Properties in Both the Directions}

For cylinders having equal properties in the two materialproperty directions, i.e., $A_{11}=A_{22}, D_{11}=D_{22}$, the expression for modal density can be written in a simple form as

$$
\begin{gathered}
n(f)=\frac{2 A f \rho_{m}}{N} \int_{0}^{\frac{\pi}{2}}\left\{\left(\frac{f_{2}}{f_{1}}+\sqrt{\left(\frac{f_{2}}{f_{1}}\right)^{2}+\frac{4 N^{2}}{f_{1}\left(\rho_{m} \omega^{2}-f_{3}\right)}}\right)-\right. \\
\left.\frac{2 N^{2}}{f_{1}\left(\rho_{m} \omega^{2}-f_{3}\right) \sqrt{\left(\frac{f_{2}}{f_{1}}\right)^{2}+\frac{4 N^{2}}{f_{1}\left(\rho_{m} \omega^{2}-f_{3}\right)}}}\right\} d \theta
\end{gathered}
$$

The functions of $\theta$ become

$f_{1}=D_{11}\left(1-2 c^{2} s^{2}+\gamma c^{2} s^{2}\right)$;

$f_{2}=D_{11}$

$f_{3}=\frac{A_{11} c^{4}\left(1-\alpha^{2}\right)}{a^{2}\left\{1-2 c^{2} s^{2}+\frac{1-\alpha^{2}-2 \alpha \beta}{\beta} c^{2} s^{2}\right\}}$;

with $\frac{A_{12}}{A_{11}}=\alpha ; \frac{A_{66}}{A_{11}}=\beta ; \frac{2\left(D_{12}+2 D_{66}\right)}{D_{11}}=\gamma$.

\section{INFLUENCE OF TRANSVERSE SHEAR DEFORMATION AND ORTHOTROPY}

\subsection{Transverse Shear}

To understand the effect of the transverse shear deformation, modal densities are obtained (in one-third octave bands) for a honeycomb sandwich composite cylinder, whose properties are given in Section 5, and the results are presented in Table 3 and Fig. 4.

Modal density of a thin cylinder increases with frequency, reaches a maximum and then converges to the modal density of flat plates. The transverse shear deformation increases modal density. The influence is negligible at low frequencies but very significant at high frequencies. At high frequencies due to the presence of transverse shear deformation modal densities increase with frequency whereas it remains constant if the transverse shear deformation is neglected. Figure 4 clearly indicates that in the absence of the present expression, modal density calculated by neglecting transverse shear can result in significant error at higher frequencies.

Modal densities, as a function of frequency, for different values of $N$ are shown in Fig. 5. Results show that modal density

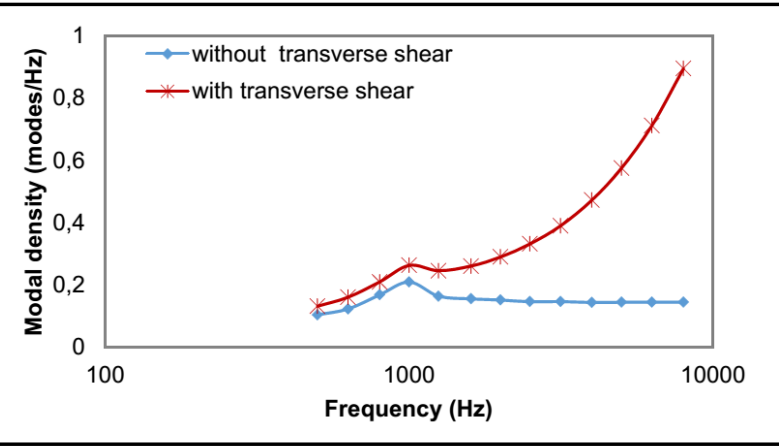

Figure 4. Modal densities of honeycomb sandwich composite cylinder.

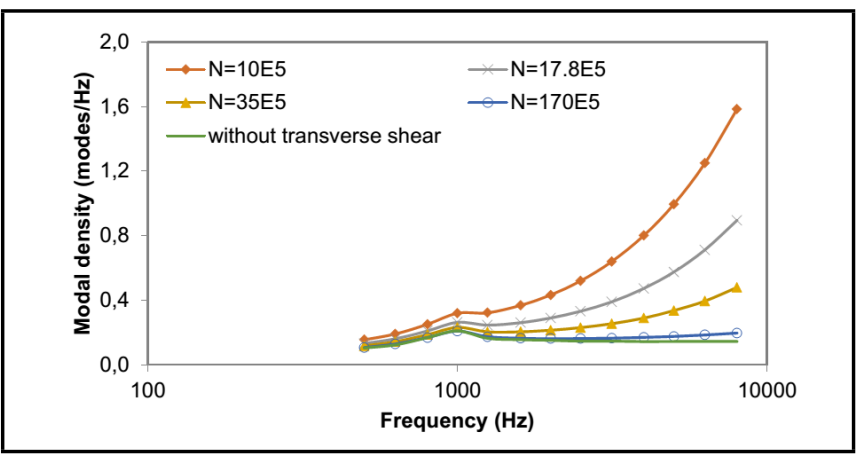

Figure 5. Effect of transverse shear flexibility on modal density of composite cylinder.

increases sharply with increase in shear flexibility and the effect is larger at high frequencies. When the shear rigidity is very high the modal densities of the cylinder are identical to those of thin cylinder.

\subsection{Orthotropic Nature of the Cylinder}

Modal density is also influenced by the in-plane shear property, apart from other factors, which is represented by the parameters $\beta$ and $\gamma$. As both the parameters have similar effects on the modal density, results for various values of $\gamma$ only are presented.

A typical cylinder whose properties are same in both the directions, given in Section 3, is considered. Modal densities for various values of $\gamma$ are shown in Fig. 6. The results show that the parameter $\gamma$ has a significant role and it has similar influence at all frequencies. Modal densities increase with decrease in the values of $\gamma$. In other words, modal densities increase with decrease in in-plane shear modulus and Poisson's ratio. For an isotropic material $\gamma=2$ and it is equal to 0.17 for the cylinder considered.

\subsection{Use of Expression for Isotropic Cylinders}

In the absence of the expression derived in this work one could use the available expression reported in the literature which is for isotropic materials. The term $D_{11}$ of the given cylinder can be equated to $D$ of the equivalent isotropic cylinder and the thickness can be worked out. Wilkinson's expres$\operatorname{sion}^{4}$ can now be used to obtain the modal densities. The cylinder whose details are given in Section 3 is considered. The isotropic cylinder is a honeycomb sandwich cylinder with the same core as the given cylinder but face sheets made of Aluminium alloy having Young's modulus $7.2 \times 10^{10} \mathrm{~N} / \mathrm{m}^{2}$. The thickness of the face sheet of the isotropic cylinder is $0.3 \mathrm{~mm}$. The results are given in Fig. 7. 


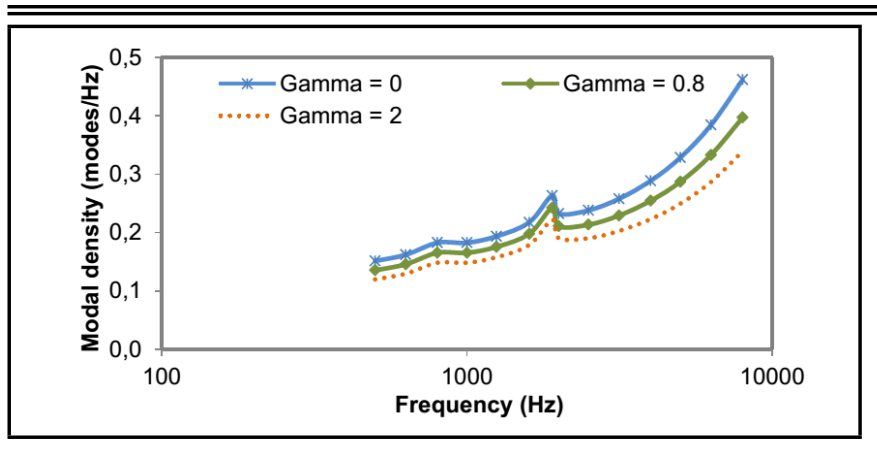

Figure 6. Effect of in-plane shear stiffness on the modal density.

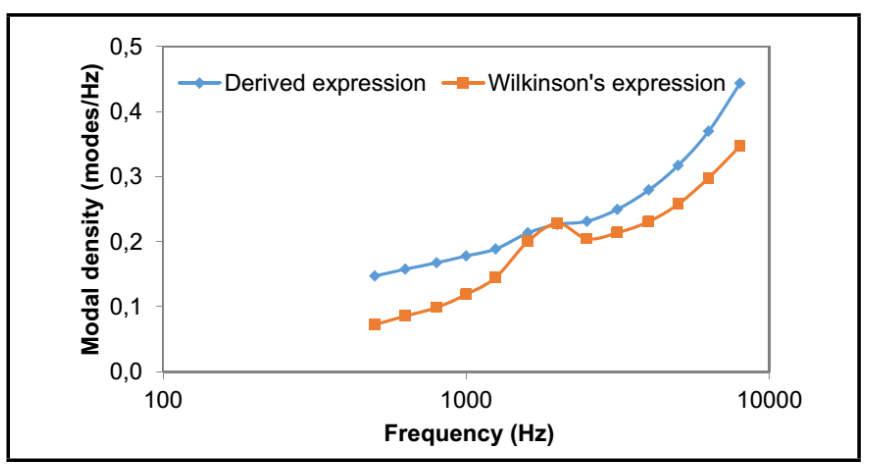

Figure 7. Modal densities estimated using expression for isotropic cylinders.

The results show that the estimated modal densities are very much lower if isotropic models are used signifying the need for the expression derived here.

\section{CONCLUSIONS}

Expressions for estimating the natural frequency, mode count and modal density of composite cylindrical shells incorporating transverse shear deformation are derived. Modal densities of typical cylinders of spacecraft are obtained. The results are in accordance with the number of modes determined using the finite element method. Transverse shear deformation increases modal density and the impact is very significant at higher frequencies. Effect of the orthotropic nature of the face sheets is to increase the modal density further but its impact is present at all frequencies. It is shown that in the absence of the expression derived here the modal densities computed will be in significant error.

\section{REFERENCES}

1 Lyon, R. H. Statistical Energy Analysis of Dynamical Systems: Theory and Applications, MIT Press, Cambridge, MA (1975).

2 Norton, M. P. Fundamentals of Noise and Vibration Analysis for Engineers, Cambridge University Press, England, (1989).

3 Xie, G., Thompson, D. J., and Jones, C. J. C. Mode count and modal density of structural systems: relationships with boundary conditions, Journal of Sound and Vibration, 274, 621-651, (2004). https://dx.doi.org/10.1016/j.jsv.2003.05.008

4 Wilkinson, J. P. D. Modal densities of certain shallow structural elements, Journal of the Acoustical Society of America, 43, 245-251, (1968). https://dx.doi.org/10.1121/1.1910773
5 Erickson, L. L. Modal densities of sandwich panels: theory and experiment, Shock and Vibration Bulletin, 39, 1-16, (1969).

6 Ferguson, N. S. and Clarkson, B. L. The modal density of honeycomb shells, Transactions of the ASME, Journal of Vibration, Acoustics, Stress and Reliability in Design, 108, 399-404, (1986). https://dx.doi.org/10.1115/1.3269362

7 Elliot, G. H. The evaluation of the modal density of paraboloidal and similar shells, Journal of Sound and Vibration, 126, 477-483, (1988). https://dx.doi.org/10.1016/0022-460x(88)90225-8

8 Finnveden, S. Evaluation of modal density and group velocity by a finite element method, Journal of Sound and Vibration, 273, 51-75, (2004). https://dx.doi.org/10.1016/j.jsv.2003.04.004

9 Farshidianfar, M. H., Farshidianfar, A., and Moghadam, M. M. Mode count and modal density of isotropic circular cylindrical shells using a modified wavenumber space integration method, Journal of Vibration and Acoustics, 136, 041004, (2014). https://dx.doi.org/10.1115/1.4027212

10 Ramachandran, P. and Narayanan, S. Evaluation of modal density, radiation efficiency and acoustic response of longitudinally stiffened cylindrical shell, Journal of Sound and Vibration, 304 (1-2), 154-174, (2007). https://dx.doi.org/10.1016/j.jsv.2007.02.020

11 Josephine Kelvina Florence, S. and Renji, K. Modal density of thin composite cylindrical shells, Journal of Sound and Vibration, 365, 157-171, (2016). https://dx.doi.org/10.1016/j.jsv.2015.11.030

12 Chun, C. K. and Dong, S. B. Shear constitutive relations for laminated anisotropic shells and plates: Part II-Vibrations of composite cylinders, Transactions of ASME, 59, 380389, (1992). https://dx.doi.org/10.1115/1.2899531

13 Bert, C. W. and Kumar, M. Vibration of cylindrical shells of bimodulus materials, Journal of Sound and Vibration, 81, 107-121, (1982). https://dx.doi.org/10.1016/0022$460 \times(82) 90180-8$

14 Qatu, M. S. Vibration of Laminated Plates and Shells, Elsevier Academic Press, UK, (2004).

15 Librescu, L., Khdeir, A. A., and Frederick, D. A shear deformable theory for laminated composite shallow shelltype panels and their response analysis I: Free vibration and buckling, Acta Mechanica, 76, 1-33, (1989). https://dx.doi.org/10.1007/bf01175794

16 Bhimaraddi, A. Free vibration analysis of doubly curved shallow shells on rectangular planform using 3-D elasticity theory, International Journal of Solids and Structures, 27, 897-913, (1991). https://dx.doi.org/10.1016/00207683(91)90023-9

17 Soedel, W. Vibration of Shells and Plates, Marcel Dekker, New York, (1981).

18 Renji, K., Nair, P. S., and Narayanan, S. Modal density of composite honeycomb sandwich panels, Journal of Sound and Vibration, 195 (5), 687-699, (1996). https://dx.doi.org/10.1006/jsvi.1996.0456 